\title{
New amide alkaloids from Piper longum fruits
}

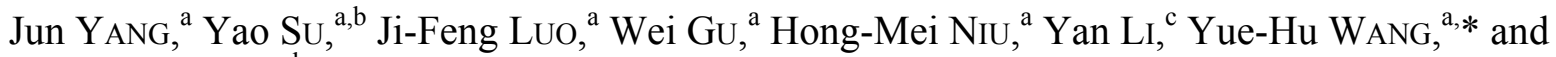 \\ Chun-Lin LONG ${ }^{\mathrm{a}, \mathrm{d}, *}$ \\ ${ }^{a}$ Key Laboratory of Economic Plants and Biotechnology, Kunming Institute of Botany, Chinese Academy of Sciences, \\ Kunming 650201, China \\ ${ }^{\mathrm{b}}$ College of Resources and Environmental Sciences, Hebei Agricultural University, Hebei 071000, China \\ ${ }^{\mathrm{c}}$ State Key Laboratory of Phytochemisty and Plant Resources in West China, Kunming Institute of Botany, Chinese \\ Academy of Sciences, Kunming 650201, China \\ ${ }^{\mathrm{d}}$ College of Life and Environmental Sciences, Minzu University of China, Beijing 100081, China
}

Received 15 September 2013; Accepted 12 November 2013

(C) The Author(s) 2013. This article is published with open access at Springerlink.com

\begin{abstract}
Three new amide alkaloids piperlongumamides A-C (1-3), together with 12 known ones (4-15), were isolated from the fruits of Piper longum. The structures of the new isolates were determined using spectroscopic data analyses. Cytotoxic activity of these amides against HL-60 (human leukemia), A-549 (human lung cancer), MCF-7 (human breast cancer), SMMC-7721 (human liver cancer) and SW480 (human rectal cancer) cell lines were evaluated. Piperchabamide B (11) exhibited weak inhibitory activity against HL-60 $\left(\mathrm{IC}_{50}=21.32 \mu \mathrm{M}\right), \mathrm{A}-549\left(\mathrm{IC}_{50}=23.82 \mu \mathrm{M}\right)$ and MCF-7 $\left(\mathrm{IC}_{50}=16.58 \mu \mathrm{M}\right)$ cell lines.
\end{abstract}

Keywords: Piperaceae, Piper longum, amide alkaloids, piperlongumamides, piperchabamide B, cytotoxicity

\section{Introduction}

Piper longum L. (Piperaceae) is a slender aromatic climber with perennial woody roots which grows primarily in tropical regions. Its fruits and roots are used to treat various disease and ailments in traditional Chinese medicinal and ethnomedicinal practice. Uses include expectorant, curing dyspepsia, sleep problems, asthma, nausea, diarrhea, lumbarleg pain and arthralgia. ${ }^{1}$ The plant mainly contains amide alkaloids which have been used with anti-hepatitis B virus (antiHBV), ${ }^{2,3}$ apoptotic, ${ }^{4}$ leishmanicidal, ${ }^{5}$ cytotoxic, ${ }^{6}$ mosquito larvicidal, ${ }^{7,8}$ phytotoxic, ${ }^{9}$ anti-inflammatory, ${ }^{10}$ antihyperlipidemic, ${ }^{11,12}$ cell adhesion inhibitory, ${ }^{13}$ antiplatelet, ${ }^{11,14}$ acylCoA: cholesterol acyltransferase (ACAT) inhibitory, ${ }^{15}$ antifungal $^{16}$ and coronary vasorelaxant activities. ${ }^{17}$ In addition to amide alkaloids, phytochemicals present also include prenylated phenolic compounds ${ }^{18}$ and aromatic esters ${ }^{19}$ are found in the plant. In our continuing research on bioactive constituents of Piper species, ${ }^{20-22}$ three new amide alkaloids piperlongumamides A-C (1-3), along with 12 previously identified ones (Figure 1) were isolated from the fruits of $P$. longum. The structural elucidation of the new compounds and the bioassay results are reported.

\footnotetext{
*To whom correspondence should be addressed. E-mail: wangyuehu@mail.kib.ac.cn (Y.H. Wang); long@mail.kib.ac.cn (C.L. Long)
}

\section{Results and Discussion}

The molecular formula of compound $1, \mathrm{C}_{22} \mathrm{H}_{31} \mathrm{NO}$, was determined using the HREIMS $\left(\mathrm{m} / z 325.2413[\mathrm{M}]^{+}\right)$, indicating eight degrees of unsaturation. Its IR spectrum showed strong absorption bands at 1722,1636 and $1452 \mathrm{~cm}^{-1}$ implying the existence of unsaturated amide and aromatic functionalities. The ${ }^{1} \mathrm{H}$ NMR and ${ }^{13} \mathrm{C}$ NMR spectra of 1 (Table 1) showed signals for one monosubstituted benzene ring $\left[\delta_{\mathrm{H}} 7.27(2 \mathrm{H}, \mathrm{m})\right.$ and $7.17(3 \mathrm{H}, \mathrm{m})]$, two conjugated trans double bonds $\left[\delta_{\mathrm{H}} 7.30\right.$ $(\mathrm{dd}, J=14.8,10.8 \mathrm{~Hz}), 6.24(\mathrm{~d}, J=14.8 \mathrm{~Hz}), 6.17$ (dd, $J=$ $15.2,10.8 \mathrm{~Hz}$ ) and $6.08(\mathrm{dt}, J=15.2,7.0 \mathrm{~Hz})]$, one carbonyl group $\left(\delta_{\mathrm{C}} 166.0\right)$ and several methylene groups. Moreover, amide alkaloids are major constituents of Piper plants, and the compound might be a phenylalkenoyl derivative. A piperidine ring was determined by the ${ }^{1} \mathrm{H}-{ }^{1} \mathrm{H}$ COSY spectrum (Figure 2). The length of the alkenoyl group was determined as 11 carbon atoms according to the molecular formula of compound $\mathbf{1}$. Finally, on the basis of the HMBC correlations (Figure 2) from $\mathrm{H}-3$ to $\mathrm{C}-1, \mathrm{H}_{2}-1^{\prime \prime}$ and $\mathrm{H}_{2}-5^{\prime \prime}$ to $\mathrm{C}-1, \mathrm{H}_{2}-10$ to $\mathrm{C}-1^{\prime}$ and $\mathrm{H}_{2}-11$ to $\mathrm{C}-2^{\prime}$ and $\mathrm{C}-6^{\prime}$, the structure of $\mathbf{1}$ was determined to be

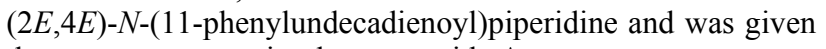
the common name piperlongumamide $\mathrm{A}$.

The molecular formula of compound $2, \mathrm{C}_{22} \mathrm{H}_{33} \mathrm{NO}$, was determined by HREIMS at $m / z 327.2554[\mathrm{M}]^{+}$. Its IR spectrum showed absorption bands at 1723,1657, 1618 and $1439 \mathrm{~cm}^{-1}$ indicating the existence of unsaturated amide and aromatic groups. Comparison of the MS and NMR data of 2 with those of 1 (Table 1), the difference was that a trans double bond at 
<smiles>O=C(/C=C/C=C/C=C/c1ccccc1)N1CCCCC1</smiles>

1<smiles>O=C(/C=C/C=C/c1ccc2c(c1)OCO2)N1CCCCC1</smiles>

4<smiles>CC(C)CNC(=O)/C=C/C=C/c1ccc2c(c1)OCO2</smiles>

7<smiles>CC(C)CNC(=O)/C=C/C=C/C=C/[13C]/C=C/c1ccc2c(c1)OCO2</smiles><smiles>CC(C)C=CC=CC(=O)N1CCCCC1</smiles>

12<smiles>O=C(/C=C/C=C/CC/C=C/c1ccc2c(c1)OCO2)N1CCCCC1</smiles><smiles>O=C(/C=C\C=C\c1ccc2c(c1)OCO2)N1CCCCC1</smiles>

5<smiles>CCC/C=C\CCCC=C/C=C/C(=O)N1CCCCC1</smiles>

3<smiles>O=C(/C=C\C=C/c1ccc2c(c1)OCO2)N1CCCCC1</smiles>

6<smiles>C=C/C=C/C=C/C(=O)NCC(C)C</smiles>

8<smiles>O=C(/C=C/CC/C=C/c1ccc2c(c1)OCO2)N1CCCCC1</smiles>

11

Figure 1. Structures of amide alkaloids 1-15 from Piper longum

C-4(5) disappeared in compound 2. Based on the ${ }^{1} \mathrm{H}-{ }^{1} \mathrm{H}$ COSY and HMBC correlations of $\mathbf{2}$ (Figure 2), the structure of $\mathbf{2}$ was deduced to be (2E)-N-(11-phenylundecenoyl)piperidine and was given the common name piperlongumamide $\mathrm{B}$.

Compound 3 has the molecular formula $\mathrm{C}_{19} \mathrm{H}_{31} \mathrm{NO}$ based on HREIMS at $m / z 289.2414[\mathrm{M}]^{+}$. The IR spectrum indicated the presence of an unsaturated amide group (1720, 1713, 1630 and $1447 \mathrm{~cm}^{-1}$ ). The ${ }^{1} \mathrm{H}$ and ${ }^{13} \mathrm{C}$ NMR spectra of 3 (Table 2) displayed signals for two trans-conjugated diene moiety $\left[\delta_{\mathrm{H}}\right.$ $6.24(1 \mathrm{H}, \mathrm{d}, J=15.0 \mathrm{~Hz}, \mathrm{H}-2), 7.29(1 \mathrm{H}, \mathrm{dd}, J=15.0,11.0 \mathrm{~Hz}$, $\mathrm{H}-3), 6.18(1 \mathrm{H}, \mathrm{dd}, J=15.0,11.0 \mathrm{~Hz}, \mathrm{H}-4)$ and $6.08(1 \mathrm{H}, \mathrm{dt}, J$ $=15.0,7.2 \mathrm{~Hz}, \mathrm{H}-5)]$, one methyl group $\left[\delta_{\mathrm{H}} 0.89(3 \mathrm{H}, \mathrm{t}, J=\right.$ $6.8 \mathrm{~Hz}, \mathrm{H}-14)]$, one carbonyl group $\left(\delta_{\mathrm{H}} 166.1\right)$ and several methylene groups. Also, signals at $\delta_{\mathrm{H}} 5.32(1 \mathrm{H}, \mathrm{m}, \mathrm{H}-9)$ and $5.38(1 \mathrm{H}, \mathrm{m}, \mathrm{H}-10)$ were due to an additional double bond, which was deduced as $Z$ configuration by the chemical shifts of the allylic carbons $\left[\delta_{\mathrm{C}} 26.8(\mathrm{C}-8) \text { and } 27.1(\mathrm{C}-11)\right]^{21,22}$ Based on above analysis and by comparison of its NMR data with those of compound $\mathbf{1}$, compound $\mathbf{3}$ was deduced as an alkenoyl piperidine. According to ${ }^{1} \mathrm{H}-{ }^{1} \mathrm{H}$ COSY correlations of 3 (Figure 2), the connections from $\mathrm{C}-1$ to $\mathrm{C}-11, \mathrm{C}-13$ to $\mathrm{C}-14$ and $\mathrm{C}-1^{\prime}$ to $\mathrm{C}-5^{\prime}$ were confirmed. Finally, based on the HMBC
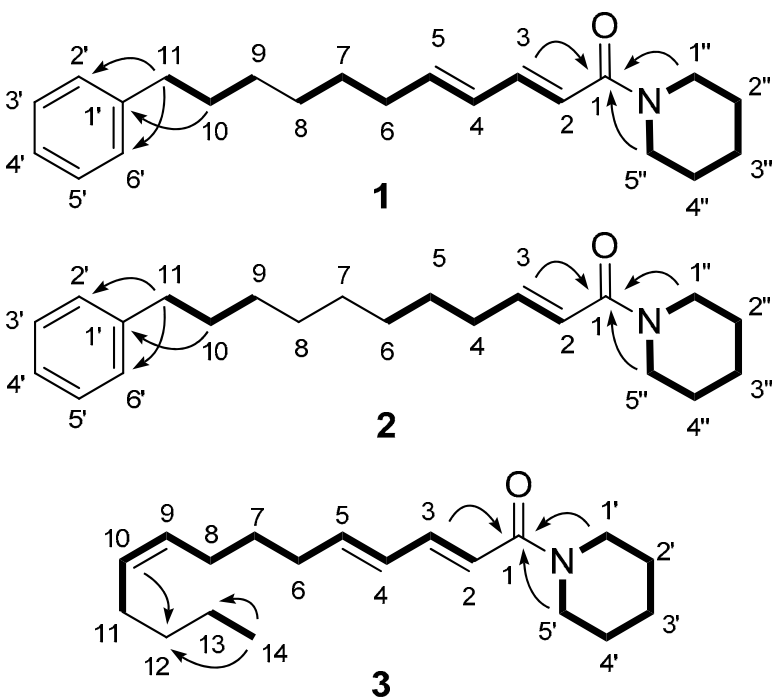

Figure 2. Key ${ }^{1} \mathrm{H}-{ }^{1} \mathrm{H}$ COSY (bold) and HMBC (arrows, $\mathrm{H} \rightarrow \mathrm{C}$ ) correlations of compounds $\mathbf{1}-\mathbf{3}$ 
Table $1 .{ }^{1} \mathrm{H}$ and ${ }^{13} \mathrm{C}$ NMR spectral data of 1 and 2 in $\mathrm{CDCl}_{3}(\delta$ in ppm)

\begin{tabular}{|c|c|c|c|c|}
\hline \multirow[b]{2}{*}{ No. } & \multicolumn{2}{|c|}{1} & \multicolumn{2}{|c|}{2} \\
\hline & $\delta_{\mathrm{C}}(150 \mathrm{MHz})$ & $\delta_{\mathrm{H}}(600 \mathrm{MHz})$ & $\delta_{\mathrm{C}}(100 \mathrm{MHz})$ & $\delta_{\mathrm{H}}(400 \mathrm{MHz})$ \\
\hline 1 & $166.0 \mathrm{C}$ & & $165.8 \mathrm{C}$ & \\
\hline 2 & $118.0 \mathrm{CH}$ & $6.24,1 \mathrm{H}, \mathrm{d}(14.8)$ & $120.2 \mathrm{CH}$ & $6.23,1 \mathrm{H}$, br. d $(15.1)$ \\
\hline 3 & $143.7 \mathrm{CH}$ & $7.30,1 \mathrm{H}, \mathrm{dd}(14.8,10.8)$ & $146.6 \mathrm{CH}$ & $6.84,1 \mathrm{H}, \mathrm{dt}(15.1,7.0)$ \\
\hline 4 & $128.9 \mathrm{CH}$ & $6.17,1 \mathrm{H}, \mathrm{dd}(15.2,10.8)$ & $32.7 \mathrm{CH}_{2}$ & $2.18,2 \mathrm{H}, \mathrm{q}$ like $(7.0)$ \\
\hline 5 & $143.3 \mathrm{CH}$ & $6.08,1 \mathrm{H}, \mathrm{dt}(15.2,7.0)$ & $28.5 \mathrm{CH}_{2}$ & $1.43,2 \mathrm{H}, \mathrm{m}$ \\
\hline 6 & $33.1 \mathrm{CH}_{2}$ & $2.14,2 \mathrm{H}, \mathrm{q}$ like $(7.0)$ & $29.5^{\mathrm{b}} \mathrm{CH}_{2}$ & $1.29,2 \mathrm{H}, \mathrm{m}$ \\
\hline 7 & $28.8 \mathrm{CH}_{2}$ & $1.41,2 \mathrm{H}, \mathrm{m}$ & $29.5^{\mathrm{b}} \mathrm{CH}_{2}$ & $1.29,2 \mathrm{H}, \mathrm{m}$ \\
\hline 8 & $29.2 \mathrm{CH}_{2}$ & $1.32,2 \mathrm{H}, \mathrm{m}$ & $29.4^{\mathrm{b}} \mathrm{CH}_{2}$ & $1.29,2 \mathrm{H}, \mathrm{m}$ \\
\hline 9 & $29.2 \mathrm{CH}_{2}$ & $1.32,2 \mathrm{H}, \mathrm{m}$ & $29.3^{\mathrm{b}} \mathrm{CH}_{2}$ & $1.29,2 \mathrm{H}, \mathrm{m}$ \\
\hline 10 & $31.6 \mathrm{CH}_{2}$ & $1.60,2 \mathrm{H}, \mathrm{m}$ & $31.6 \mathrm{CH}_{2}$ & $1.60,2 \mathrm{H}, \mathrm{m}$ \\
\hline 11 & $36.0 \mathrm{CH}_{2}$ & $2.59,2 \mathrm{H}, \mathrm{t}(7.8)$ & $36.1 \mathrm{CH}_{2}$ & $2.59,2 \mathrm{H}, \mathrm{t}(7.7)$ \\
\hline $1^{\prime}$ & $142.9 \mathrm{C}$ & & $143.0 \mathrm{C}$ & \\
\hline $2^{\prime}, 6^{\prime}$ & $128.4 \mathrm{CH}$ & $7.27,2 \mathrm{H}, \mathrm{m}$ & $128.3 \mathrm{CH}$ & $7.27,2 \mathrm{H}, \mathrm{m}$ \\
\hline $3^{\prime}, 5^{\prime}$ & $128.5 \mathrm{CH}$ & $7.17,2 \mathrm{H}, \mathrm{m}$ & $128.5 \mathrm{CH}$ & $7.18,2 \mathrm{H}, \mathrm{m}$ \\
\hline $4^{\prime}$ & $125.7 \mathrm{CH}$ & $7.17,1 \mathrm{H}, \mathrm{m}$ & $125.7 \mathrm{CH}$ & $7.18,1 \mathrm{H}, \mathrm{m}$ \\
\hline $1^{\prime \prime}$ & $45.2^{\mathrm{a}} \mathrm{CH}_{2}$ & $3.57,2 \mathrm{H}, \mathrm{m}$ & $42.9^{\mathrm{a}} \mathrm{CH}_{2}$ & $3.54,2 \mathrm{H}, \mathrm{m}$ \\
\hline $2^{\prime \prime}$ & $26.3 \mathrm{CH}_{2}$ & $1.58,2 \mathrm{H}, \mathrm{m}$ & $26.2 \mathrm{CH}_{2}$ & $1.56,2 \mathrm{H}, \mathrm{m}$ \\
\hline $3^{\prime \prime}$ & $24.7 \mathrm{CH}_{2}$ & $1.65,2 \mathrm{H}, \mathrm{m}$ & $24.8 \mathrm{CH}_{2}$ & $1.65,2 \mathrm{H}, \mathrm{m}$ \\
\hline $4^{\prime \prime}$ & $26.3 \mathrm{CH}_{2}$ & $1.58,2 \mathrm{H}, \mathrm{m}$ & $26.2 \mathrm{CH}_{2}$ & $1.56,2 \mathrm{H}, \mathrm{m}$ \\
\hline $5^{\prime \prime}$ & $45.2^{\mathrm{a}} \mathrm{CH}_{2}$ & $3.56,2 \mathrm{H}, \mathrm{m}$ & $46.9^{\mathrm{a}} \mathrm{CH}_{2}$ & $3.54,2 \mathrm{H}, \mathrm{m}$ \\
\hline
\end{tabular}

${ }^{a}$ Detected by HSQC spectrum. ${ }^{b}$ Data under the same entry are exchangeable.

correlations (Figure 2) from $\mathrm{H}-3, \mathrm{H}_{2}-1^{\prime}$ and $\mathrm{H}_{2}-5^{\prime}$ to $\mathrm{C}-1, \mathrm{H}-10$ to $\mathrm{C}-12, \mathrm{Me}-14$ to $\mathrm{C}-12$ and $\mathrm{C}-13$, the structure of 3 was determined to be $(2 E, 4 E, 9 Z)-N$-tetradecatrienoylpiperidine and was given the common name piperlongumamide $\mathrm{C}$.

The known compounds were identified as piperine (4), ${ }^{23}$ isopiperine (5), ${ }^{24}$ chavicine $(\mathbf{6}),{ }^{24}$ piperlonguminine (7), ${ }^{25}$ pellitorine $(\mathbf{8}),{ }^{26}$ brachystamide B $(\mathbf{9}),{ }^{27}$ guineensine $(\mathbf{1 0}),{ }^{25}$ piperchabamide $\mathrm{B}(\mathbf{1 1}),{ }^{28}(2 E, 4 E)$ - $N$-dodecadienoylpiperidine (12), ${ }^{29}$ dehydropipernonaline (13), ${ }^{12}$ pipernonaline $(\mathbf{1 4})^{16}$ and piperolein $\mathrm{B}(\mathbf{1 5})^{12}$ by comparison of their NMR and MS data with those reported in the literature.

In a previous study, we found that an amide alkaloid 1[(9E)-10-(3,4-methylenedioxyphenyl)-9-decenoyl]pyrrolidine from $P$. boehmeriaefolium was cytotoxic. ${ }^{21}$ Therefore, all of the amides from $P$. longum were evaluated for their inhibitory activities against HL-60 (human leukemia), A-549 (human lung cancer), MCF-7 (human breast cancer), SMMC-7721 (human liver cancer) and SW480 (human rectal cancer) cell lines. Piperchabamide B (11) exhibited weak inhibitory activity against HL-60 cell line $\left(\mathrm{IC}_{50}=21.32 \mu \mathrm{M}\right), \mathrm{A}-549\left(\mathrm{IC}_{50}=\right.$ $23.82 \mu \mathrm{M})$ and MCF-7 $\left(\mathrm{IC}_{50}=16.58 \mu \mathrm{M}\right)$ (Table 3). Other tested compounds were inactive.

\section{Experimental Section}

General Experimental Procedures. UV spectra were recorded on a Shimadzu double-beam 210A spectrometer (Shimadzu Co., Shimadzu, Japan). IR spectra were recorded on a Bruker Tensor 27 Fourier transform infrared spectrometer (Bruker, Karlsruhe, Germany) with $\mathrm{KBr}$ pellets. ESIMS and HREIMS analyses were carried out on an API Qstar-Pulsar-1 mass spectrometer (Applied Biosystems/MDS Sciex, Ontario, Canada) and Waters AutoSpec Premier P776 (Waters, Milford, USA), respectively. ${ }^{1} \mathrm{H}$ and ${ }^{13} \mathrm{C}$ NMR spectra were collected on a Bruker AM-400, DRX-500 and Avance III-600 spectrometers (Bruker Bio-Spin GmbH, Rheinstetten, Germany) with TMS as an internal standard. Semi-preparative HPLC was performed on an Agilent 1200 series pump (Agilent Technologies, Santa Clara, USA) equipped with a diode array detector and a Zorbax SB- $\mathrm{C}_{18}$ column $(5.0 \mu \mathrm{m}, \phi 9.4 \times 250$ $\mathrm{mm}$ ). Both analytical and preparative TLC conductedusing
Table 2. ${ }^{1} \mathrm{H}(600 \mathrm{MHz})$ and ${ }^{13} \mathrm{C}$ NMR (150 MHz) spectral data of 3 in $\mathrm{CDCl}_{3}(\delta$ in ppm)

\begin{tabular}{ccl}
\hline No. & \multicolumn{1}{c}{$\delta_{\mathrm{C}}$} & \multicolumn{1}{c}{$\delta_{\mathrm{H}}$} \\
\hline 1 & $166.1 \mathrm{C}$ & \\
2 & $117.8 \mathrm{CH}$ & $6.24,1 \mathrm{H}, \mathrm{d}(15.0)$ \\
3 & $144.1 \mathrm{CH}$ & $7.29,1 \mathrm{H}, \mathrm{dd}(15.0,11.0)$ \\
4 & $129.1 \mathrm{CH}$ & $6.18,1 \mathrm{H}, \mathrm{dd}(15.0,11.0)$ \\
5 & $143.4 \mathrm{CH}$ & $6.08,1 \mathrm{H}, \mathrm{dt}(15.0,7.2)$ \\
6 & $32.6 \mathrm{CH}_{2}$ & $2.16,2 \mathrm{H}, \mathrm{q}$ like $(7.2)$ \\
7 & $28.9 \mathrm{CH}_{2}$ & $1.48,2 \mathrm{H}, \mathrm{m}$ \\
8 & $26.8 \mathrm{CH}_{2}$ & $2.04,2 \mathrm{H}, \mathrm{m}$ \\
9 & $129.1 \mathrm{CH}$ & $5.32,1 \mathrm{H}, \mathrm{m}$ \\
10 & $130.7 \mathrm{CH}$ & $5.38,1 \mathrm{H}, \mathrm{m}$ \\
11 & $27.1 \mathrm{CH}_{2}$ & $2.01,2 \mathrm{H}, \mathrm{m}$ \\
12 & $32.0 \mathrm{CH}_{2}$ & $1.31,2 \mathrm{H}, \mathrm{m}$ \\
13 & $22.5 \mathrm{CH}_{2}$ & $1.31,2 \mathrm{H}, \mathrm{m}$ \\
14 & $14.2 \mathrm{CH}_{3}$ & $0.89,3 \mathrm{H}, \mathrm{t}(6.8)$ \\
$1^{\prime}$ & $45.2^{\mathrm{a}} \mathrm{CH}_{2}$ & $3.56,2 \mathrm{H}, \mathrm{m}$ \\
$2^{\prime}$ & $26.3 \mathrm{CH}_{2}$ & $1.58,2 \mathrm{H}, \mathrm{m}$ \\
$3^{\prime}$ & $24.7 \mathrm{CH}_{2}$ & $1.66,2 \mathrm{H}, \mathrm{m}$ \\
$4^{\prime}$ & $26.3 \mathrm{CH}_{2}$ & $1.58,2 \mathrm{H}, \mathrm{m}$ \\
$5^{\prime}$ & $45.2^{\mathrm{a}} \mathrm{CH}_{2}$ & $3.56,2 \mathrm{H}, \mathrm{m}$ \\
\hline
\end{tabular}

${ }^{\mathrm{a}}$ Detected by HSQC spectrum.

silica gel plates $\left(\mathrm{GF}_{254}\right.$, Yantai Institute of Chemical Technology, Yantai, China). The spots were initially visualized using UV light (254 and $366 \mathrm{~nm}$ ) and subsequently visualised by spraying a solution of $5 \% \mathrm{H}_{2} \mathrm{SO}_{4}$ onto the TLC plate, which was subsequently heated. Column chromatography was performed using silica gel (80-100 mesh and 300-400 mesh; Qingdao Makall Group Co., Ltd., Qingdao, China), $\mathrm{C}_{18}$ silica gel (40-75 $\mu \mathrm{m}$, Fuji Silysia Chemical, Ltd., Kasugai, Japan) and Sephadex LH-20 (GE Healthcare Bio-Xciences AB, Uppsala, Sweden).

Plant Material. The fruits of $P$. longum were purchased from Yikan Chinese Herbal Medicine Ltd., Qujing, China, in October 2011. The plant material was identified by Dr. Guang-Wan $\mathrm{Hu}$, at Kunming Institute of Botany, Chinese Academy of Sciences. A voucher specimen (QJ1101) has been deposited at Key Laboratory of Economic Plants and Biotechnology, Kunming Institute of Botany.

\section{黑 Springer}


Table 3. Cytotoxicity of piperchabamide B (11) from Piper longum*

\begin{tabular}{|c|c|c|c|c|c|}
\hline \multirow[b]{2}{*}{ Compound } & \multicolumn{5}{|c|}{$\mathrm{IC}_{50}(\mu \mathrm{M})$} \\
\hline & HL-60 & SMMC-7721 & A-549 & MCF-7 & SW480 \\
\hline Piperchabamide B (11) & 21.32 & $>40$ & 23.82 & 16.58 & $>40$ \\
\hline Cisplatin (positive control) & 1.32 & 6.24 & 11.83 & 15.17 & 12.95 \\
\hline Taxol (positive control) & $<0.008$ & $<0.008$ & $<0.008$ & $<0.008$ & $<0.008$ \\
\hline
\end{tabular}

*Other tested compounds were inactive.

Extraction and Isolation. The dried powdered fruits $(20 \mathrm{~kg})$ of $P$. longum were extracted using $\mathrm{MeOH}$ (4, 3 and $3 \mathrm{~h}$, resp.) under reflux. The combined $\mathrm{MeOH}$ extracts were evaporated under reduced pressure to yield a residue, which was suspended in $\mathrm{H}_{2} \mathrm{O}$ and then partitioned successively with petroleum ether and $\mathrm{CHCl}_{3}$ to produce two corresponding portions. After TLC testing, the two portions were combined as both contained alkaloids. The combined extract $(1377 \mathrm{~g})$ was subjected to column chromatography over silica gel G (80-100 mesh) using petroleum ether/EtOAc $(1: 0 \rightarrow 0: 1)$ to yield ten fractions (Fr. A-J) and also compound 4 (1.8 g).

Fr. D (19 g) was separated using column chromatography $\left(\mathrm{C} 18, \mathrm{MeOH} / \mathrm{H}_{2} \mathrm{O}, 80: 20\right)$ to give $8(56.2 \mathrm{mg})$. Fr. E (12 g) was partitioned by column chromatography $\left(\mathrm{C} 18, \mathrm{MeOH} / \mathrm{H}_{2} \mathrm{O}\right.$, $60: 40 \rightarrow 95: 5)$ to produce fractions (E1-E7). Fr. E1 (1.1 g) was fractionated by column chromatography (Sephadex LH-20, $\mathrm{MeOH}$; silica gel, petroleum ether/ $\left.\mathrm{Me}_{2} \mathrm{CO}, 1: 1\right)$ and prep. TLC (petroleum ether/EtOAc, 10:1) to give $5(20.0 \mathrm{mg}), \mathbf{6}$ $(27.0 \mathrm{mg})$ and $7(8.2 \mathrm{mg})$.

Fr. E3 (2.5 g) was fractionated by column chromatography (Sephadex LH-20, MeOH; silica gel, petroleum ether/ $\mathrm{Me}_{2} \mathrm{CO}$, $3: 1)$ and semi-preparative HPLC $\left(\mathrm{MeOH} / \mathrm{Me}_{3} \mathrm{CN} / \mathrm{H}_{2} \mathrm{O}\right.$, $50: 35: 15,4 \mathrm{~mL} / \mathrm{min})$ to obtain $13\left(23.8 \mathrm{mg}, t_{R}=5.995 \mathrm{~min}\right)$ and $14\left(37.3 \mathrm{mg}, t_{R}=6.818 \mathrm{~min}\right)$. Fr. E4 was fractionated by column chromatography (Sephadex LH-20, MeOH) to give two parts (E4a and E4b). Fr. E4a (233.6 mg) was subjected to by column chromatography (silica gel, petroleum ether/ $\mathrm{Et}_{2} \mathrm{NH}$, 50:1) and semi-preparative HPLC $\left(\mathrm{MeCN} / \mathrm{H}_{2} \mathrm{O}, 90: 10,3\right.$ $\mathrm{ml} / \mathrm{min})$ to give $12\left(1 \mathrm{mg}, t_{R}=11.984 \mathrm{~min}\right), \mathbf{1}\left(2.1 \mathrm{mg}, t_{R}=\right.$ $12.858 \mathrm{~min}), 2$ ( $\left.4 \mathrm{mg}, t_{R}=16.182 \mathrm{~min}\right), \mathbf{3}\left(8.4 \mathrm{mg}, t_{R}=13.959\right.$ min). Fr. E4b (743.7 mg) was isolated repeatedly by column chromatography (silica gel, petroleum ether $/ \mathrm{Me}_{2} \mathrm{CO}, 5: 1$; petroleum ether/EtOAc, 4:1; petroleum ether/ $\left.\mathrm{Et}_{2} \mathrm{NH}, 10: 1\right)$ to give $10(17.2 \mathrm{mg})$ and $\mathbf{1 1}(18.0 \mathrm{mg})$, and a remaining fraction, which was purified by semi-preparative $\mathrm{HPLC}\left(\mathrm{MeCN} / \mathrm{H}_{2} \mathrm{O}\right.$, $60: 40,4 \mathrm{~mL} / \mathrm{min})$ to give $\mathbf{1 5}\left(3.5 \mathrm{mg}, t_{R}=17.027 \mathrm{~min}\right)$ and $\mathbf{1 1}$ (4 mg, $\left.t_{R}=28.529 \mathrm{~min}\right)$. Fr. E5 $(1.3 \mathrm{~g})$ was fractionated by column chromatography (Sephadex LH-20, $\mathrm{MeOH}$ ) and prep. TLC $\left(\mathrm{CHCl}_{3} /\right.$ EtOAc, $\left.10: 1\right)$ to give $9(23.9 \mathrm{mg})$.

Piperlongumamide A (1): pale yellow oil; UV (MeOH): $\lambda_{\max }(\log \varepsilon) 205(2.77) \mathrm{nm}$. IR (KBr) $v_{\max } 1722,1636,1452$, $1254,748,699,571 \mathrm{~cm}^{-1} .{ }^{1} \mathrm{H}$ and ${ }^{13} \mathrm{C}$ NMR spectral data see Table 1. ESIMS (positive) $m / z 326[\mathrm{M}+\mathrm{H}]^{+}$; HREIMS: $m / z$ $325.2413[\mathrm{M}]^{+}$(calcd for $\mathrm{C}_{22} \mathrm{H}_{31} \mathrm{NO}, 325.2406$ ).

Piperlongumamide B (2): pale yellow oil; UV (MeOH): $\lambda_{\max }(\log \varepsilon) 209(2.93) \mathrm{nm}$. IR (KBr) $v_{\max } 1723,1657,1618$, 1439, 1219, 1069, 1023, 974, 903, $637 \mathrm{~cm}^{-1} .{ }^{1} \mathrm{H}$ and ${ }^{13} \mathrm{C}$ NMR spectral data see Table 1. ESIMS (positive) $\mathrm{m} / z 350[\mathrm{M}+\mathrm{Na}]^{+}$; HREIMS: $m / z 327.2554[\mathrm{M}]^{+}$(calcd for $\mathrm{C}_{22} \mathrm{H}_{33} \mathrm{NO}, 327.2562$ ).
Piperlongumamide C (3): pale yellow oil; UV (MeOH): $\lambda_{\max }(\log \varepsilon) 207(2.33) \mathrm{nm}$. IR (KBr) $v_{\max } 1720,1713,1630$, $1447,1253,1229,1166,1082,1022,978,800,588 \mathrm{~cm}^{-1} .{ }^{1} \mathrm{H}$ and ${ }^{13} \mathrm{C}$ NMR spectral data see Table 2. ESIMS (positive) $\mathrm{m} / \mathrm{z}$ $290[\mathrm{M}+\mathrm{H}]^{+}$; HREIMS: $\mathrm{m} / z$ 289.2414 [M] ${ }^{+}$(calcd for $\left.\mathrm{C}_{19} \mathrm{H}_{31} \mathrm{NO}, 289.2406\right)$.

MTS Assay for Cytotoxicity. The isolated amide alkaloids were tested in vitro for their cytotoxicity against proliferation of human leukemia HL-60 cell line, human lung cancer A-549, human breast cancer MCF-7, human liver cancer SMMC-7721 and human rectal cancer SW480 using the MTS assay.

The 3-(4,5-dimethylthiazol-2-yl)-5(3-carboxymethoxyphenyl)-2-(4-sulfopheny)-2H-tetrazolium (MTS) (Promega, Beijing, China) is a mix-based cell titer assay and was performed as previous described. ${ }^{30}$ Initially, cells in their log-phase of their cycle were seeded in 96-well plates (500010000 cells/well, NEST Biotechnology, Wuxi, China) using a standard $100 \mu \mathrm{L}$ volume, with paired single cell suspension containing $10 \%$ fetal bovine serum (DMEM or RMPI1640, Thermo Fisher Scientific, Beijing, China). The cell were further treated with indicated concentrations of the compounds dissolved in DMSO, which were set the regular thickness $40 \mu \mathrm{M}$ for the initial screening and five concentrations of each compound were fixed in the concentration compounds inhibited tumor cell growth by approximately $50 \%$ to achieve a total culture medium in a volume of $200 \mu \mathrm{L}$. After incubation for $48 \mathrm{~h}$ at $37^{\circ} \mathrm{C}$, a $20 \mu \mathrm{L}$ of MTS solution and $100 \mu \mathrm{L}$ DMEM were added into the well, incubation was continued for another 1-4 h. The absorbance was measured at the detection wavelength of $490 \mathrm{~nm}\left(L_{1}\right)$ and the reference wavelength of $680 \mathrm{~nm}$ $\left(L_{2}\right)^{31}$ and cytotoxicity for each compound was expressed as $\mathrm{IC}_{50}$ values.

\section{Electronic Supplementary Material}

Supplementary material is available in the online version of this article at http://dx.doi.org/10.1007/s13659-013-0073-0 and is accessible for authorized users.

\section{Acknowledgments}

This work was funded by the Natural Science Foundation of Yunnan Province, China (No. 2011FZ205), the National Natural Science Foundation of China (Nos. 31070288, 31161140345), the Ministry of Education of China through its 111 and 985 projects (Nos. B08044, MUC98506-01000101 \& MUC985-9), and the Japan Society for the Promotion of Science (No. JSPS/AP/109080).

Open Access This article is distributed under the terms of the Creative Commons Attribution License which permits any use,

\section{算 Springer}


distribution, and reproduction in any medium, provided the original author(s) and source are credited.

\section{References}

[1] Editorial Board of 'Zhonghua Bencao', State Administration of Traditional Chinese Medicine of the People's Republic of China, Zhonghua Bencao; Shanghai Scientific and Technical Publishing House: Shanghai, 1999, Vol. 3, p. 434.

[2] Jiang, Z. Y.; Liu, W. F.; Zhang, X. M.; Luo, J.; Ma, Y. B.; Chen, J. J. Bioorg. Med. Chem. Lett. 2013, 23, 2123-2127.

[3] Jiang, Z. Y.; Liu, W. F.; Huang, C. G.; Huang, X. Z. Fitoterapia 2013, 84, 222-226.

[4] Lee, W.; Kim, K. Y.; Yu, S .N.; Kim, S. H.; Chun, S. S.; Ji, J. H.; Yu, H. S.; Ahn, S. C. Biochem. Biophys. Res. Commun. 2013, 430, 406-412.

[5] Ghosal, S.; Deb, A.; Mishra, P.; Vishwakarma, R. Planta Med. 2012, 78, 906-908.

[6] Mishra, P.; Sinha, S.; Guru, S.; Bhushan, S. K.; Vishwakarma, R. A.; Ghosal, S. J. Asian Nat. Prod. Res. 2011, 13, 143-148.

[7] Madhu, S. K.; Vijayan, V. A.; Shaukath, A. K. Asian Pac. J. Tropical Med. 2011, 4, 112-116.

[8] Yang, Y. C.; Lee, S. G.; Lee, H. K.; Kim, M. K.; Lee, S. H.; Lee, H. S. J. Agric. Food Chem. 2002, 50, 3765-3767.

[9] Huang, H. Z.; Morgan, C. M.; Asolkar, R. N.; Koivunen, M. E.; Marrone, P. G. J. Agric. Food Chem. 2010, 58, 9994-10000.

[10] Lee, S. W.; Kim, M. S.; Park, M. H.; Park, S. J.; Lee, W. S.; Chang, J. S.; Rho, M. C. Bull. Korean Chem. Soc. 2010, 31, 921-924.

[11] Jin, Z.; Borjihan, G.; Zhao, R.; Sun, Z.; Hammond, G. B.; Hammond, T. Phytother. Res. 2009, 23, 1194-1196.

[12] Lee, S. W.; Rho, M. C.; Park, H. R.; Choi, J. H.; Kang, J. Y.; Lee, J. W.; Kim, K.; Lee, H. S.; Kim, Y. K. J. Agric. Food Chem. 2006, 54, 9759-9763.

[13] Lee, S. W.; Kim, Y. K.; Kim, K.; Lee, H. S.; Choi, J. H.; Lee, W S.; Jun, C. D.; Park, J. H.; Lee, J. M.; Rho, M. C. Bioorg. Med. Chem. Lett. 2008, 18, 4544-4546.

[14] Iwashita, M.; Oka, N.; Ohkubo, S.; Saito, M.; Nakahata, N. Eur. J. Pharmacol. 2007, 570, 38-42.

[15] Lee, S. W.; Rho, M. C.; Nam, J. Y.; Lim, E. H.; Kwon, O. E.; Kim, Y. H.; Lee, H. S.; Kim, Y. K. Planta Med. 2004, 70, 678679.
[16] Lee, S. E.; Park, B. S.; Kim, M. K.; Choi, W. S.; Kim, H. T. Cho, K. Y.; Lee, S. G.; Lee, H. S. Crop Prot. 2001, 20, 523-528.

[17] Shoji, N.; Umeyama, A.; Saito, N.; Takemoto, T.; Kajiwara, A.; Ohizumi, Y. J. Pharm. Sci. 1986, 75, 1188-1189.

[18] Ohno, O.; Watabe, T.; Nakamura, K.; Kawagoshi, M.; Uotsu, N.; Chiba, T.; Yamada, M.; Yamaguchi, K.; Yamada, K.; Miyamoto, K. Biosci. Biotechnol. Biochem. 2010, 74, 1504-1506.

[19] Kumar, S.; Arya, P.; Mukherjee, C.; Singh, B. K.; Singh, N.; Parmar, V. S.; Prasad, A. K.; Ghosh, B. Biochem. 2005, 44, 15944-15952.

[20] Liu, H. X.; Chen, K.; Sun, Q. Y.; Yang, F. M.; Hu, G. W.; Wang, Y. H.; Long, C. L. J. Nat. Prod. 2013, 76, 732-736.

[21] Tang, G. H.; Chen, D. M.; Qiu, B. Y.; Sheng, L.; Wang, Y. H.; Hu, G. W.; Zhao, F. W.; Ma, L. J.; Wang, H.; Huang, Q. Q.; Xu, J. J.; Long, C. L.; Li, J. J. Nat. Prod. 2011, 74, 45-49.

[22] Yang, S. X.; Sun, Q. Y.; Yang, F. M.; Hu, G. W.; Luo, J. F.; Wang, Y. H.; Long, C. L. Planta Med. 2013, 79, 693-696.

[23] Olsen, R. A.; Spessard, G. O. J. Agric. Food Chem. 1981, 29, 942-944.

[24] Hashimoto, K.; Yaoi, T.; Koshiba, H.; Yoshida, T.; Maoka, T.; Fujiwara, Y.; Yamamoto, Y.; Mori, K. Food Sci. Technol. Int. 1996, 2, 24-29.

[25] Lee, S. A.; Hwang, J. S.; Han, X. H.; Lee, C.; Lee, M. H.; Choe, S. G.; Hong, S. S.; Lee, D.; Lee, M. K.; Hwang, B. Y. Arch. Pharmacal Res. 2008, 31, 679-683.

[26] Yasuda, I.; Takeya, K.; Itokawa, H. Chem. Pharm. Bull. 1981 29, 564-566

[27] Abad Martínez, M. J.; Benito, P. B. Stud. Nat. Prod. Chem. 2005, 30, 393-418.

[28] Morikawa, T.; Matsuda, H.; Yamaguchi, I.; Pongpiriyadacha, Y.; Yoshikawa, M. Planta Med. 2004, 70, 152-159.

[29] Shakhmaev, R. N.; Ishbaeva, A. U.; Zorin, V. V. Russ. J. Org. Chem. 2012, 48, 908-913.

[30] Yang, X.; Yuan, D. D.; Jiang, X. J.; Xi, Z. J. J. Peking Univ. (Health Sci.). 2013, 45, 221-226.

[31] Ghafar, S. A. A.; Ismail, M.; Yazan, L. S.; Fakurazi, S.; Ismail N.; Chan, K. W.; Tahir, P. M. Evid-Based Complement. Altern. Med. 2013, 2013, 1-8. 